\title{
CHALLENGES AND ENABLERS OF AUGMENTED REALITY TECHNOLOGY FOR IN SITU WALKTHROUGH APPLICATIONS
}

SUBMITTED: August 2019

REVISED: October 2019

PUBLISHED: January 2020 at https://www.itcon.org/2020/3

EDITOR: Amor R.

DOI: $10.36680 /$ j.itcon.2020.003

\section{Ivan Mutis}

Assistant Professor, Civil and Architectural Engineering, Director of the iConSenSe Laboratory, Illinois Institute of Technology, Chicago, USA

imutissi@iit.edu

\author{
Abhijeet Ambekar \\ Graduate Student, Computer Science, Illinois Institute of Technology, Chicago, USA \\ aambekar@hawk.iit.edu
}

SUMMARY: The successful implementation of situational-awareness enhancement using next-generation augmented reality in construction project walkthroughs has significant associated challenges. In this research, an augmented reality (AR) approach was developed to evaluate and showcase the trade-offs between capacity, latency, and reliability for the technology. The approach, named "i-Tracker", overlays visualizations of designs by creating virtual content to be superimposed into physical contexts. $i$-Tracker uses the latest generation of mobile computing technology to effectively locate design information from existing parametric engineering designs (architectural, structural, and mechanical objects) and create a fully animated scene in situ. The i-Tracker technology uses a combination of depth-sensing cameras and inertial measurement unit (IMU) sensors. The evaluation of this technology demonstrates the requirements and limitations applicable to the implementation of this technology in job sites.

In the example use case, the device's relative position and orientation with respect to the user are estimated. Performance features such as motion tracking, localization, error dispersion with respect to luminance, system processing speed, and ambiguity in the feature tracking are evaluated. $i$-Tracker contributes to the body of literature and current work on the use of positioning and tracking systems in real construction sites within the AR context. This project advances understanding of rapid implementations and the use of AR visualizations on job sites, utilizing significant progress in mobile and ubiquitous computing with faster central processing units (CPUs) and graphical processing units (GPUs).

KEYWORDS: Indoor Motion Tracking, Augmented Reality, 2D Feature Tracking, Localization

REFERENCE: Ivan Mutis, Abhijeet Ambekar (2020). Challenges and enablers of augmented reality technology for in situ walkthrough applications. Journal of Information Technology in Construction (ITcon), Vol. 25, pg. 5571, DOI: $10.36680 /$ j.itcon.2020.003

COPYRIGHT: (C) 2020 The author(s). This is an open access article distributed under the terms of the Creative Commons Attribution 4.0 International (https://creativecommons.org/licenses/by/4.0/), which permits unrestricted use, distribution, and reproduction in any medium, provided the original work is properly cited. 


\section{INTRODUCTION}

Designs and construction sites are unique due to their varying local conditions - characteristics that result in increased complexity in decision-making activities and lead to a high degree of freedom in project engineers' and managers' choice of actions. The variation and a significant number of changing (or "ill-structured" (Jonassen, 2011)) conditions in construction sites hinder individuals' abilities to effectively associate designs to the physical project context. Information technologies have been used as a mediating mechanism to facilitate association-tasks of designs to the project context (Mutis, Fruchter, \& Menassa, 2018). Augmented Reality (AR) technology has been widely used as an enhancing and interacting mechanism to experience the user's surrounding in construction site environments (Alsafouri \& Ayer Steven, 2019; Hou, Wang, \& Truijens, 2013; Koch, Neges, König, \& Abramovici, 2014; Park \& Kim, 2013; Zollmann et al., 2014). At the forefront of the emergence of applications of these new technologies, a significant amount of research has been undertaken (Rankohi \& Waugh, 2013; P. Wang, Wu, Wang, Chi, \& Wang, 2018; X. Wang, Kim, Love, \& Kang, 2013) to inform and contribute to the incorporation of AR applications in the construction industry. One such application is for increasing the efficacy of in situ-walkthroughs, which can be described as a pre-planned or organized process of reviews in the job site that is based on the stakeholders' project needs. Though the potential for benefit in this application exists, there are significant challenges that must be surmounted for successful integration. The challenges center mostly on location-tracking that enables an accurate location and position of augmented visualizations of design components in the construction site. Addressing these challenges will critically empower project stakeholders, learners, and all job-site personnel to have more compelling immersive experiences with AR technology for engineering design interpretations during walkthroughs.

An approach, named "i-Tracker," that investigated the AR technology functionality for its implementation for construction site walkthroughs is presented herein. $i$-Tracker focuses on research that combines location-tracking information (relative motion from the origin) with information about objects located in the physical environment. The goal is to provide an enhanced level of perception and movement and an additional level of immersion, thereby allowing users to better navigate in situ and better interpret the application of individual design components for construction projects. The immersive aspects of $i$-Tracker focalize on better matching the visual experience with feedback of self-motion and body position produced with stimuli of the user's body segment (e.g., hand, head) (motor-sensory modality), and on accommodating visual and motor-sensory modality with high-fidelity orientation in the physical space.

This work contributes to advance the understanding of AR functionality in walkthroughs by raising awareness of the technical limitations for the implementation of the technology and paving the way for new building-technology scaffolded solutions to enable in situ interpretation of complex engineering designs. $i$-Tracker employs state-ofthe-art technology to produce visualizations of superimposed, computer-generated objects. The presented work gives a high-level view of design implementation and discusses the challenges that exist in high-performance applications. Ultimately, this research contributes to enhancing the knowledge and understanding of AR implementation practices related to in situ walkthroughs and its applications in order to improve AR applications in the construction industry. Improved understanding of AR implementation complexities will - among other benefits - reduce user effort necessary for its operation, it will simplify the application of the technology, and it will limit the extent of corrections needed during operation. The research informs the construction, architecture, and engineering community with methods to improve practices when implementing AR technology to enable an immersive experience that reaches beyond lab-based prototype-technology.

This paper has four major components: (1) description and benefits of using AR for walkthroughs on the job site; (2) presentation of a brief background on VR and AR applications, including previous work on AR overlay developments on construction sites; (3) the challenges and opportunities of AR technology as points of departure, which were used as foundational factors for the technical evaluation, and evaluation factors for in situ operations, including assessments from experiments using a case study example; and (4) discussion on trade-off factors between technology performance, functionality, and reliability.

\section{AWARENESS OF IN SITU WALKTHROUGHS USING AR TECHNOLOGY}

The use of augmented reality representations of project designs is widespread in industry. This type of representations is virtual, 3D objects that allow users to map designs to the physical context (Woo, 2007) to, 
therefore, identify conditions and constraints of the construction process or construction-management-related activities (Zaher, Greenwood, \& Marzouk, 2018). Due to the difficulty in deploying the computing technology used for design visualization in situ, project personnel still struggle to relate virtual, 3D representations of designs to physical site conditions. This lack of awareness may impair anticipation of problems in the execution of construction processes (e.g., clashes of design components) or within construction management activities themselves. For example, when planning the installation process for pre-cast columns (an element of a structural engineering system), it is necessary for personnel to understand the order and organization of components according to the constraints and conditions found on the job site. This can be facilitated by overlaying the visualization of the design in situ. As a result of its immersive capabilities, mobile-usage ability, and high costbenefit impact, AR technology is an ideal medium to promote job-site technology usage. An example is the technology's ability to superimpose visualizations from the design onto specific locations at the job site. Effective implementation of the technology would enhance situational awareness, enable the user to trace, and visit augmentations. and, ultimately, infer the components' functionalities within the design system or constraints within a construction process.

Situational awareness through AR has multiple beneficial outcomes, including the training of workers and learners to track the progress of designs in situ. AR enhances awareness by increasing engineering design cognition through a computer-generated representation. It improves design interpretation skills by transferring critical contextual issues (e.g., the location of materials, the functionality of temporary structures, and the installation sequence of design components) from a physical context into an AR environment. The underlying theoretical basis of situation awareness within job sites can be framed by spatial-temporal ability principles (Mutis \& Issa, 2014).

Individuals' abilities to relate spatial and temporal information is labeled their spatial-temporal cognitive ability (Antonenko \& Mutis, 2017; Mutis, 2018). Spatial information defines how construction resources are related to one another in a contextual space, and temporal information specifies the order, sequences, and hierarchies of resources in a process. Spatial-temporal ability enhances users' (e.g., construction personnel) systematic understanding of designs in the job site, including coordination among engineering systems, construction resources (i.e., materials, parts, and equipment), processes (e.g., erecting a steel structure), and organizational aspects of a project (e.g., management of multiple trades in a construction activity). This understanding requires integration of spatial and temporal information, especially construction management practices. Spatial-temporal ability involves relating digital representations of designs (i.e., engineering designs) to the physical context. A well-developed spatial-temporal ability enables workers to - based on spatial-temporal information - identify, process, and comprehend information about construction management activities in situ. The perception of in situ elements and events concerning time or space, and the associated comprehension of their meanings for planning activities, is an example.

AR technology use during walkthroughs should assist personnel in developing a comprehensive in situ situational awareness. This awareness impacts personnel's understanding of the visualizations of designs, including other forms of representation of information of designs, such as text-based information (e.g., specification of products). Recent trends in AR-technology development incorporate sensors, mobile computing and wireless features, which enable construction of technology environments that allow users to simultaneously view: (1) location tracking, using an indoor position system for location awareness and navigation management; (2) spatially-located design components, using computer-generated 3D objects; and (3) text-based representations of designs (see Figure 1).
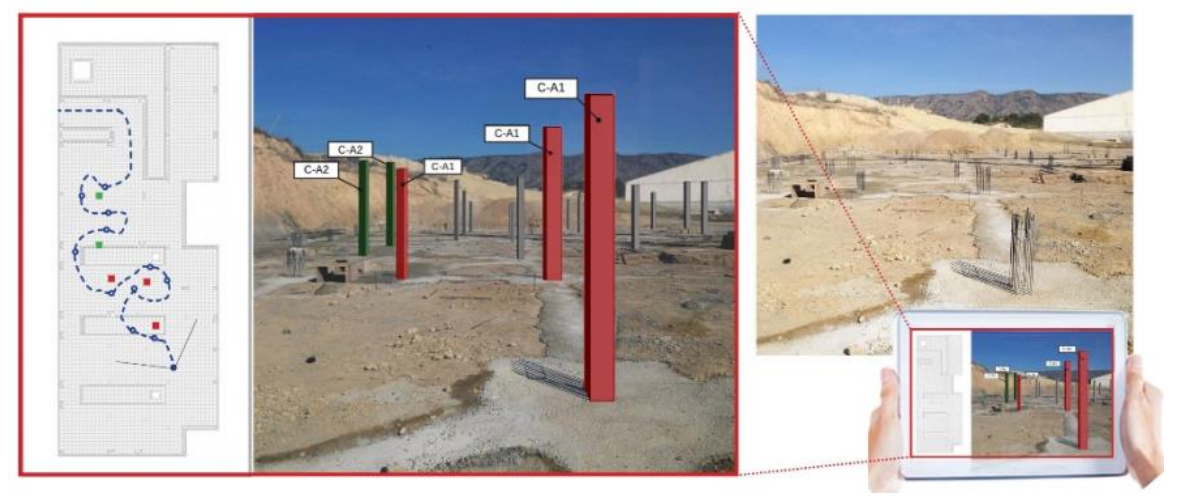

FIG. 1: AR technology applied to an in situ walkthrough. 


\section{BACKGROUND}

\subsection{Augmented reality and limiting factors for practical use}

AR technology combines virtual objects and real-world observations. Virtual objects not only come into view as part of the user's environment, but users can also interact with the objects (R. T. Azuma, 1997). In simple words, AR is a technology that involves the overlay of computer graphics in the real world. Typical AR systems comprise technologies, such as computing hardware (wearable device, mobile phones, tablets), motion tracking hardware (accelerometer, gyroscope), and an event registration interface (Papagiannakis, Singh, \& Magnenat-Thalmann, 2008). AR applications can be categorized as marker-based or image-localization-based systems (R. T. Azuma, 1997). Marker-based AR relies on locational information embedded in the physical world to track the user, while image-based AR systems extract physical features from the viewing frame to maintain an index of physical location references (Jianbo \& Tomasi, 2000).

AR is sometimes mistaken with the concept of mixed reality (MX), which refers to a multi-axis spectrum of areas that cover VR, AR, and telepresence (Milgram \& Kishino, 1994). The VR concept is related to a fully synthetic simulation of an environment, where users experience a high level of immersion. For example, the fundamental telepresence purpose is to extend the operator's sensory-motor and problem-solving abilities to a remote environment. The environment provides the space for the operator's presence and their interactions on the remote site in a natural way. AR is a technology that sits between VR and telepresence, as shown in Figure 2. AR systems are designed with two crucial functional components: (1) a combined tracking of the motion of real-world and virtual objects; and (2) the ability to interact with computer-generated virtual objects. AR has been deployed using various technologies, including displays, tracking, registration, and calibration. For example, for interfacing and visualization, head-mounted displays (HMDs) provide users with imagery in front of their eyes for AR overlay through a transparent display. Multiple technologies exist to provide overlays and projections, such as handheld displays and projection displays using gaming consoles.

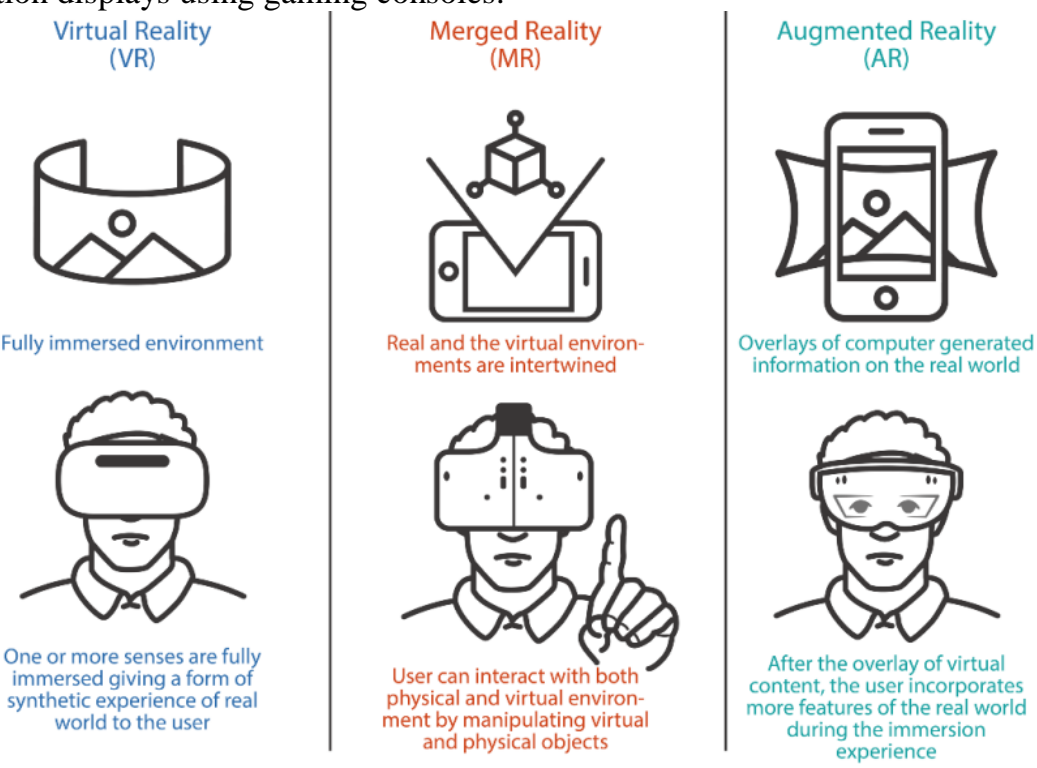

FIG. 2: Immersion features of VR, MR, and AR (Azuma 1997, Azuma, Baillot et al. 2001).

Due to recent advances in technology, many new AR application concepts have been created, including assembly guides, online shopping, project planning, and maintenance (R. Azuma et al., 2001; R. T. Azuma, 1997), to name a few. For these concepts, the typical AR design focuses on user interaction to provide realistic and rich AR experiences including detection, registration, and alignment with the real world. For example, images of objects are detected by a camera, and the relative positioning and location of the device is estimated by mapping the image data to the surrounding environment. Virtual objects are then registered within the physical location using estimated information before they are then combined with real-world objects.

When combining the real world and virtual objects, optical and video technology are key design elements that affect performance. Each of these technologies has some trade-offs on factors including resolution, flexibility, field of view, and object interaction strategies (Papagiannakis et al., 2008). Display technology can be a limiting 
factor in the development of AR systems. For example, there are no see-through displays that have sufficient brightness, resolution, field of view, and contrast to enable linking of the real-world images with computergenerated objects, and new advancements in these technologies are not yet sufficiently developed to successfully overcome these functional limitations. Furthermore, there are other limiting factors for practical use and implementation, such as size, weight, and cost (van Krevelen \& Poelman, 2010). An additional functional, limiting factor is a motion-tracking inaccuracy. Overlaid visualization on real-world scenes must be accurately positioned and aligned to reduce latencies on visual perceptions within the co-existence of the virtual and real world. In practice, the requirement of the degree of accuracy for motion-tracking functionality differs from industry to industry. For example, object inspection in museums does not require very high levels of accuracy as opposed to medical applications, where simulation of AR features demands a very high degree of accuracy and precision, for good reason.

\subsection{AR applications for design interpretation within architecture and construction engineering}

AR has been used to incorporate worksite information for the planned project into the real-world (physical context) (Hou et al., 2013) or construction job-site environment. The ability to incorporate information can be very useful for applications where workers need to process complex information to document and monitor the construction progress of a project (Zollmann et al., 2014). To monitor and control the progress of tasks and perform comparisons between as-built and as-planned project data, the responsible project control engineers require quick access to readily comprehensible project information (Zaher et al., 2018). AR systems feature a more comprehensive model to track progress and compare various milestones of the project. Zollmann et al. (2014) presented an approach to address this by presenting a model that superimposed progress data and as-planned data onto the user's view of the physical environment.

In the work proposed by Ying Zhou and Hanbin Luo (2017), the AR technology was used to perform project control activities on the job site. Their approach developed an in situ application to rapidly inspect segments' displacement of designs during tunneling construction. The system used a Building Information Modeling (BIM)based baseline model, which is overlaid on physical construction components in the job site to determine possible deviations (displacements) and to assure the quality and safety of the project. The work also included an AR-based quality inspection in real-time to reduce costs. However, although the work is a valuable contribution to AR application in project control tasks, analysis of errors in the augmentation was not performed. This analysis is critical as it directly influences the quality of visualization experiences for the AR tasks.

There is a significant amount in the literature on learning aspects using AR technology in construction engineering, architecture, and construction management (Rankohi \& Waugh, 2013; X. Wang et al., 2013). Developments focus on a wide range of AR experiences for training and learning in classrooms through the use of visual information relevant to the pedagogical material (Green \& Gilbert, 1995). For example, Shirazi and Behzadan Amir (2015) presented a collaborative context-aware mobile augmented reality tool to propose an enhancement to the construction and civil engineering curriculum. The tool was tested in a collaborative and interactive environment to enable learning experiences. Data analysis showed that AR technology had a measurable and positive impact on student learning both in the short and long term. Results also indicated that students found AR technology to be motivating, thereby contributing to the body of evidence suggesting that AR can enable a learner to gain more in-depth and long-lasting knowledge than a traditional teaching approach. Other training applications include coaching workers to develop skills to assemble products and understand safety standards and equipment operation on site (W. Li, Nee, \& Ong, 2017; X. Li, Yi, Chi, Wang, \& Chan, 2018).

Despite the advancement of AR technology, its diffusion and incorporation into training and educational practices in operator manipulation and interpretation of the representation of design (Behzadan \& Kamat, 2006; W. Li et al., 2017; P. Wang et al., 2018) are limited. For example, the use of 2D paper-based representation in lieu of virtual models still permeates the interpretation of design practices in the job site. Well-trained personnel in situ are required to reduce interpretation errors from the paper-based visualizations. The user visualization AR interface engages construction project stakeholders into a new set of practices for an enhanced awareness for problemsolving and decision-making in design interpretation (Alsafouri \& Ayer Steven, 2019). A successful introduction of such technology within job-site practices can not only provide an awareness of 3D spatial interpretation of designs in the physical space but can also assist in planning activities of the overall project (Park, Lee, Kwon, \& Wang, 2013). 


\section{ENABLERS AND LIMITATIONS OF AR TECHNOLOGY FOR WALKTHROUGHS}

The flow of advancements in AR technology can be viewed from the perspective of technology enablers and technology requirements for successful implementation. Factors of technology performance, functionality, and reliability are defining features in this perspective. These factors are balanced for technology design and implementation by imposing stringent constraints in some factors to achieve high performance in others. The following is a description of the performance, functionality, and reliability factors that informed the design of $i$ Tracker technology for efficient immersive experiences and its evaluation.

\subsection{Technology requirements and challenges for an immersive in situ walkthrough experience}

The requirements to bring more pervasive immersive experiences stemmed from management of computing, distribution and communication resources, expected quality of the use-technology interactions, and the number of running applications for user-interaction. The challenge, therefore, is determining how to manage or trade-off the computing and distribution and communication resources with an acceptable balance of performance, functionality, and reliability for a satisfactory immersive experience.

AR-technology design judiciously incorporated these trade-off factors in its deployment. The design of $i$-Tracker focused on the optimization, or balance, of remote and local computation (performance), aspects of communication (reliability), and latency of the visualizations (functional). For example, the balance of local processing on the device and that of back-end systems - with regular synchronization between the client and back-end systemswas a balance of performance, reliability, and functionality. Other trade-offs were, due to the need of a fast process of data, the use of cloud computing services to ubiquitously store and process data to preserve sensing and interactive capabilities of the mobile device by using local computing resources (Kovachev, Yu, \& Klamma, 2012), vs. local computing using the device. These trade-offs are commonly considered using a cost model (Elbamby, Perfecto, Bennis, \& Doppler, 2018), considering, for example, an application running time, network bandwidth, and battery level, for evaluation of the technology. The balancing actions among the trade-off factors considered in this cost model are data demand (performance) together with latency (functionality) and failure modes (reliability).

Data demand refers to the capacity of real-time processing responses to the interactivity of the AR applications. Capacity is quantified by bandwidth and spectral efficiency for data-rate transmissions. It enables and limits design efforts on the use of computing resources for immersive experiences. To reduce the high demand for computational power in the mobile device, trade-offs between offloading computing tasks to the cloud or to servers and network capability need to be considered. The trade-off was between the capability to meet data demands for interactivity with existing bandwidths.

Latency refers to computing and communication delays that limit aspects of immersion (e.g., matching visual experience with a sense of self-movement and segments of body positions such as hands and arms- when using the AR device). Contributing factors to delay-effects include (Elbamby et al., 2018) frame-rendering computing delay, sensor-sampling delay, and network delay. The trade-offs estimate the latency budget for an effective immersive experience.

Failure modes refer to reduced error, dropouts, and outages for an un-detached and continuous immersive experience. Robustness of the computing and network systems and application design demands techniques to reduce lags, dropouts, and drifting on computer-generated overlays or augmentations. Trade-offs among the allocation of resources in the system and requirements of the networks were designed to meet reliability constraints.

\section{2 i-Tracker AR technology approach}

The $i$-Tracker technology and its implementation were used to evaluate performance, functionality, and reliability factors against the posited challenges for walkthroughs. Using a mobile computing device, $i$-Tracker integrated BIM as visualizations of designs with features of the physical context within the real-world environment of construction sites. The aim was to gain situational awareness while providing indoor positioning and a view of the overlaid objects in the construction project. The system architecture was divided into three major building blocks, as shown in Figure 3. 


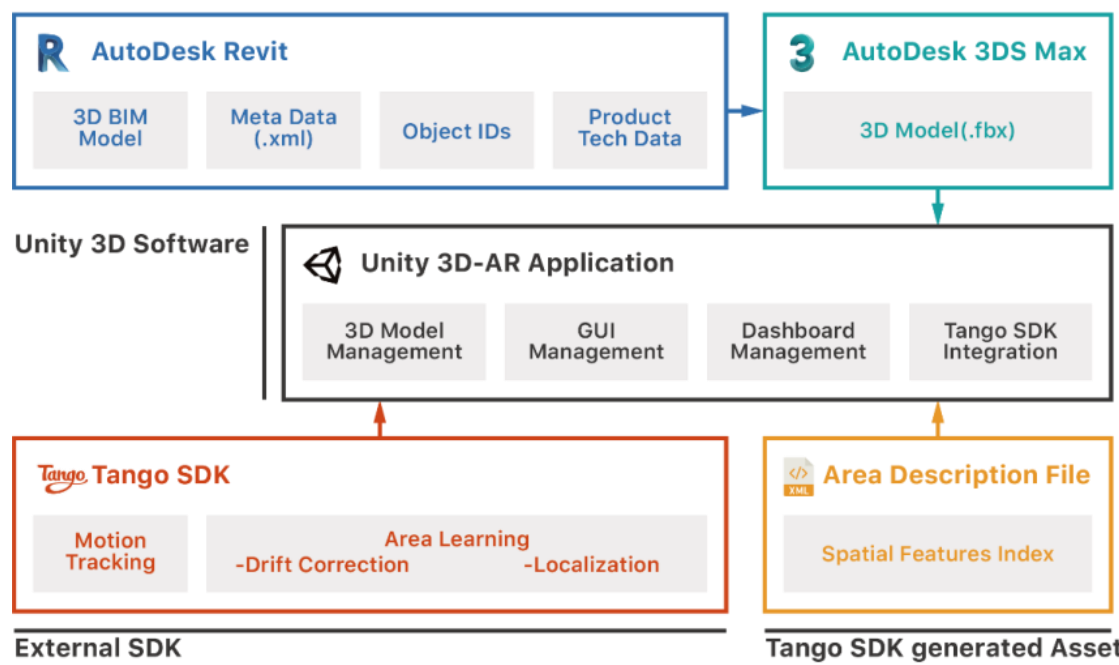

FIG. 3: System building blocks and overall development transitions.

Components of the architecture were integrated in the following phases for deployment, as outlined in Figure 4:

(1) Phase 1 - BIM model integration into AR visualizations features;

(2) Phase 2 - Feature extraction and indexing for virtual object localization, alignment and positioning;

(3) Phase 3 - Improving augmentation accuracy (error corrections); and

(4) Phase 4-Augmentation in the physical environment (use case).
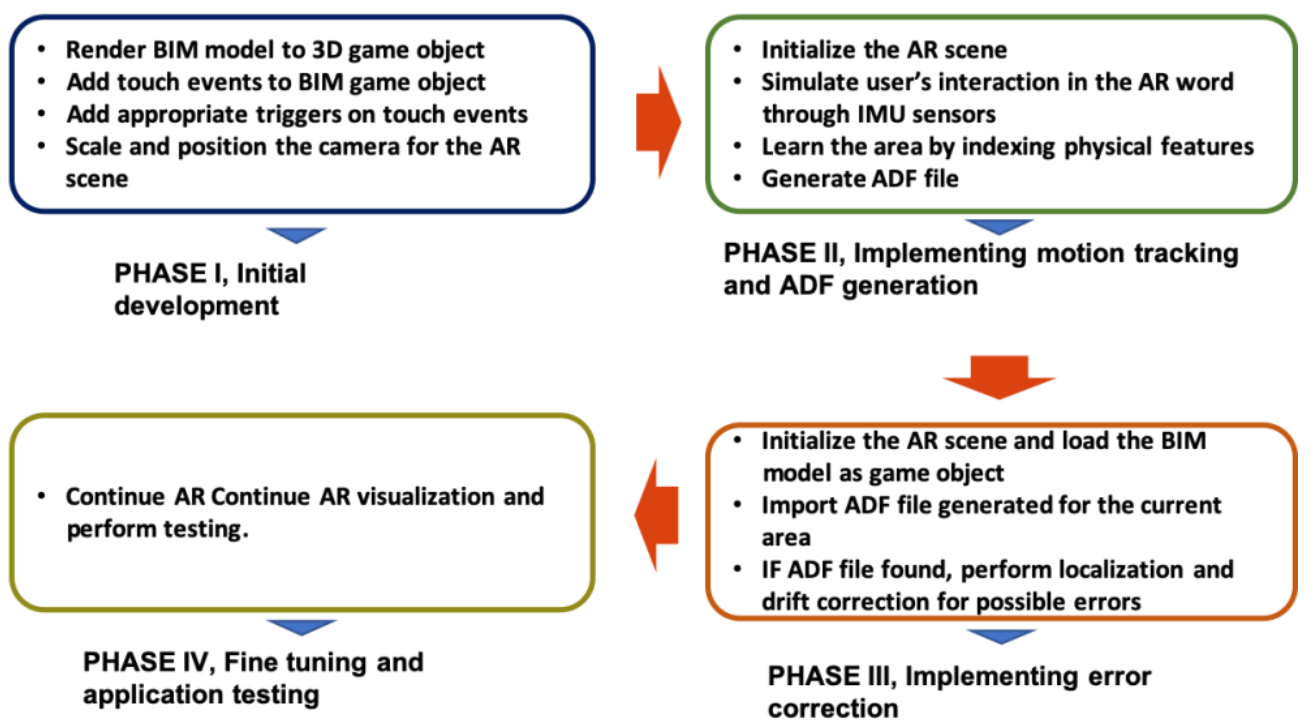

FIG. 4: Flowchart of i-Tracker development.

i-Tracker used Autodesk products for external 3D-asset processing, Unity 3D (Unity Technologies, 2018) platform for AR-object production, and the Project Tangoтм (2015) mobile device for deployment and subsequent experimentation.

\subsection{Phase 1 - BIM model integration into $A R$ visualization features}

Using a game-based engine development platform, Unity3D (Unity Technologies, 2018), BIM data was processed to associate physical features with virtual objects of the BIM design. Associating physical properties of the context into virtual objects creates features for augmentations and visual overlays. The workflow to pipeline BIM model data to the game-based engine platform was as follows: the BIM was exported from a proprietary file format (e.g., .rvt) to an interoperable standard for visual studio applications, such as fbx file format. The fbx file was imported into the project's asset folder inside Unity3D. BIM data was the input for the game engine objects. After the conversion was executed, BIM was referenced as a "game object". 
The game engine facilitated the creation of associations of physical properties to each individual object of the design. The BIM objects' notation represented a 3D construction of the game model, which generated a range of visualizations using the engine according to assigned typical physical properties of the project site (e.g., light intensity level and light-source orientation). The additional physical property was the spatial dimension, defined by the object's border. This property was linked to the game objects using an invisible layer for defining object borders, called "colliders". The effect was association of a spatial dimension to the virtual object in the physical space for either user interaction or any user-application requirement. Therefore, every time a game object was in proximity to other game objects, the colliders of both game objects informed a collision. In the same way, these colliders also helped to design user-interface detection of touch input. Thus, for instance, when a user touched a game object on the mobile device, the collision triggered the collider of that game object. These virtual colliders were attached to event detection functions to provide them with a specific task to perform an action. The associations of physical properties were executed for the entire design of the project.

\subsection{Phase 2 - Feature extraction and indexing for virtual object localization and alignment and positioning}

The design of the $i$-Tracker approach for indoor localization and positioning of augmented objects within a jobsite environment relies on motion tracking and spatial feature extraction for virtual object localization and error correction. Motion tracking, along with area learning techniques, allows a device to determine its position and motion, with error correction, as it moves through an area in physical space. After the area of interest is scanned, the extracted features are stored to be later retrieved and associated with 3D virtual objects by the system. The result is the correct alignment of the virtual objects in the physical environment. The steps of the positioning and localization process are elaborated as follows:

Motion tracking. Motion tracking is the process of accurately measuring the position and orientation of the observer's location in space. Without accurate motion tracking and registration, the superimposed virtual objects will not appear in the correct location and time, which reduces the level of immersion and co-existence with real objects. To create the immersive effect, the 3D game objects and associated text are projected into the user's view as a superimposed layer of the real world in real-time.

i-Tracker's motion tracking is a feature that connects the virtual with the physical experience, thereby generating an immersive effect. It enables AR overlays of engineering design elements in the physical space, by capturing location information to match the design object registration (3D design object) in the physical environment. Through motion tracking features of the mobile computing device's video/optical see-through displays, perception through its field of view and further immersion within the job site is possible by physically aligning the virtual design using computer-generated sensory input and using the device pose data (Barfield \& Caudell, 2001) (i.e., immersion is possible using motion tracking and pose data by matching the visual experience with the feedback of the user's body-segment position and movement and by spatially orienting and accommodating visual and motor stimuli in the physical space). Positioning data informs mobile device pose (physical position and orientation in a Euclidian space) in relation to a reference point. The pose information in AR is a feature that enhances a user's sense of spatial information (Shelton, 2003). A combination of one view of the design with the user's pose in a real environment optimizes cognitive processing (Klatzky, Wu, \& Stetten, 2008; Tang, Owen, Biocca, \& Mou, 2003; X. Wang \& Dunston, 2006). The optimization materializes when the user must integrate multiple levels of information (text and virtual images) in the immersive environment. Pose estimation data is output from inertial measurement unit (IMU) sensors, which typically comprise a three-axis accelerometer and a three-axis gyroscope (six degrees of freedom), equipped to mobile computing devices. Using these sensors, it is possible to monitor change to any of all six degrees of freedom.

In markerless AR applications, alignments are dependent on tracking the real world by estimating 3D pose accurately-unlike marker-based AR systems, which use targets. The viewing pose is considered as a six degree of freedom (6DOF) measurement: three degrees of freedom for the position and three for orientation. The tracked viewing pose defines projection of the $3 \mathrm{D}$ game object into the real-world image, so tracking accuracy determines the accuracy of the alignment. The mobile device, equipped with a camera, captures the scene frame by frame in markerless applications, with the elements in the frame acting as landmarks. $i$-Tracker markerless AR applications use a vision-based system, which relies mainly on the camera and data from the mobile device IMU sensor in combination with visual features. Error correction (e.g., local planar rectification compensated spherical distortions from images of the fish-eye lens, and predictions, using rotation data from the IMU sensor, 
compensated for fast head/hand rotations and predictions of image feature positions) was considered for the alignments.

Motion tracking is an approach that uses highly efficient 3D tracking methods based on three components: (1) Tracking initialization, (2) 2D feature tracking, and (3) 3D structure from motion (SFM) pose estimation.

Tracking initialization. Setting the reference point, where a set of most-noticeable 2D intensity corners are detected in the first image of the sequence, is the initial step for the localization. These 2D features are then tracked throughout the image sequence by local feature matching with the Kanade-Lucas-Tomasi (KLT) feature tracker operator. To further facilitate $2 \mathrm{D}$ matching, the $3 \mathrm{D}$ camera rotation velocity is measured by the inertial rotation sensor, and rotation is compensated in the images.

Feature-based vision systems perform better when salient features are registered and tracked from frame to frame. The challenge is not tracking the selected features, but tracking their association with physical points in the world (Shi \& Tomasi, 2000). For $i$-Tracker, the most-noticeable 2D intensity corners, such as those of transparent glass windows, were considered. With small inter-frame displacements, a window can be tracked by optimizing some matching criteria with respect to translation (Anandan, 1989) and linear image deformation, possibly with adaptive window size (Marr, Poggio, \& Ullman, 1979). Feature windows are selected on the measure of texture, such as a high standard deviation in the spatial intensity profile (Aggarwal \& Cai, 1999), the presence of zero crossings of the Laplacian of image intensity (Marr et al., 1979), and corners. In the 2D feature tracking method, the patterns of image intensities change in a complex way; however, away from occluding boundaries and near-surface markings, these changes can often be described as image motion.

2D features tracking to 3D structure from motion (SFM) pose estimation. From the given 2D features of the previous step, a SFM approach can be applied to estimate the metric camera pose and 3D feature positions simultaneously. Fish-eye cameras led to more-robust performance due to their larger field of view. The camera field of view covered a vast scene-area, and moving objects included only a small part of the scene, which tends to be static. The fish-eye camera, with a hemispherical view configuration, also helps to reduce jerky rotation movements since a wide view is incorporated, and a full field of view stabilizes the pose estimation (Davison, Cid, \& Kita, 2004). For perspective cameras with a small field of view, a move toward the optical axis is always illdefined because the camera moves toward the focus of contraction (FOC) — only the motion perpendicular to the FOC can be estimated reliably. In a spherical image, image position is always perpendicular to the FOC; hence, estimation of the camera motion is more reliable.

\subsection{Phase 3 - Improving augmentation accuracy (error corrections)}

The platform used to implement $i$-Tracker (Project Tangoтм) had shortcomings of accuracy in alignment of the augmentations in relation to the reference point (origin). When using IMU sensors for motion tracking, augmentations are subjected to drifting error effects. Environmental sensitivity due to temperature and noise in dynamic actions generate IMU errors. These errors accumulate and impact velocity and position calculations of the device in the physical space. The IMU-based motion-tracking approach reduces the capability of users to capture real-world features, which limits augmentation accuracy over time and the user's immersion levels. The effect felt by the user is that of having a poor situational awareness experience.

To reduce issues of accuracy within the implementation, a technique named "area learning" was employed. Area learning is an approach that captures features from the real world through depth-sensing cameras (e.g. corners, edges, and other important features) by identifying salient features in physical space and using them to estimate the position of the user. The approach serves as a mechanism to reduce drifting errors. The mechanism has two basic steps: drift correction, and localization, and is explained as follows:

Drift Correction. Drift correction is performed by loop closures using visual features, index metadata of 2D features, and location coordinates, which are stored in area description files (ADFs), to improve the accuracy of the augmentations (see Figure 5). ADF provides a searchable feature index to accurately position and orient itself in the AR world. The mobile device tracks the user's movement along with visual features found in the physical space. Such tracking enables it to determine if the area in the current frame has been previously visited or not. When the device detects previously visited features, a loop closure is triggered. ADF is used for point mapping calculation. For example, the user movement is registered in the file from a pre-determined geo-referenced, Euclidean coordinate $(\mathrm{x}, \mathrm{y}, \mathrm{z})$ (a point of reference in the construction site). The registered path is adjusted when it differs from the real trajectory of the user, using a process named "localization". 


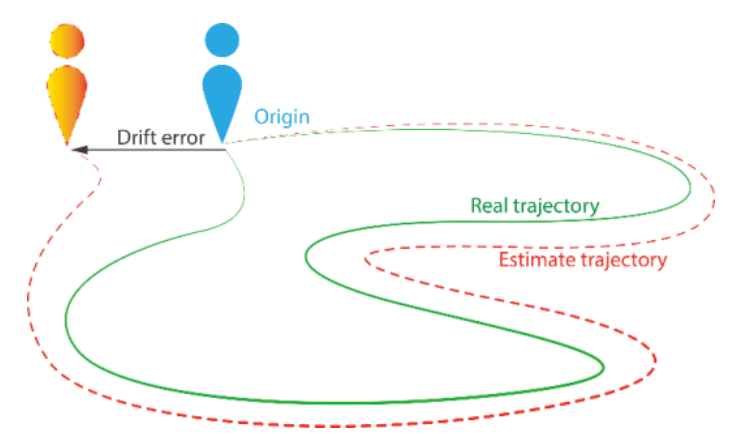

FIG. 5: Drift correction illustration.

Localization. When the user walks through a certain area, the system registers all visual features in the ADF and estimates the position relative to the origin in the file. Data of this file is further used to position the virtual game object (augmentation layer) to the user view. ADF data, together with depth-sensing camera data, accurately position the device/user position in the real world (see Figure 6).

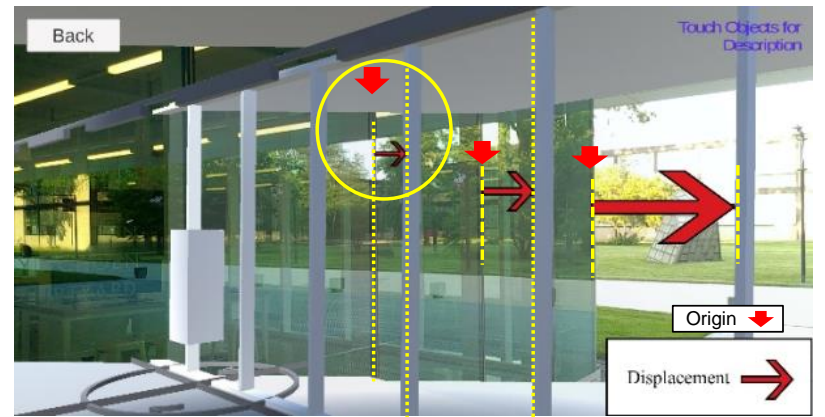

a) Observed 3D view of drifting errors on augmentations from the origin in the i-Tracker field of view.

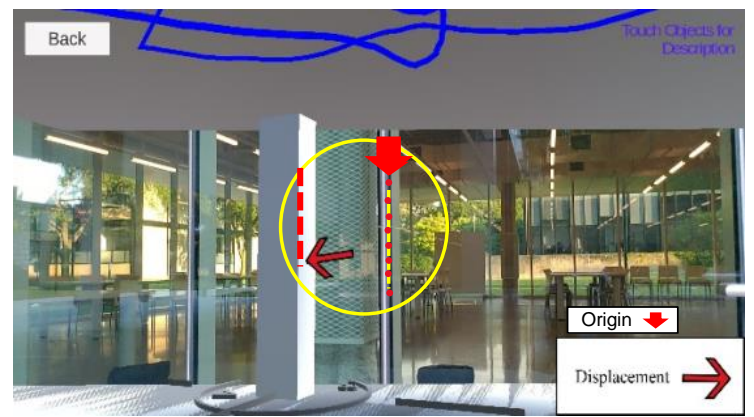

b) Reduced, observed 3D view of drifting error from origin after error-correction implementation in the i-Tracker field of view.

FIG. 6: Drifting errors (shown as bordered red arrows) that led to erroneous localization of augmentations (displacement after walkthroughs).

\section{USE CASE: I-TRACKER WALKTHROUGH IN SITU}

A use case is presented to evaluate the technical performance of the $i$-Tracker design during on-site walkthroughs. The case study informs trade-off factors (functionality, performance, and reliability) for the AR technology used and serves to validate the approach to demonstrate how these trade-off factors are balanced-imposing stringent constraints in some factors to achieve high performance in others - to provide a satisfactory immersive AR experience in walkthroughs.

Project site. The experiments took place in an educational construction project site that has a two-story, 70,000 square-foot layout with rectangular plans and top-level cantilevers - helping to shade its glazed walls - over the lower floor. It was designed with a dynamic façade of ETFE (Ethylene Tetrafluoroethylene) foil cushions.

Instruments. The researchers used the project's BIM to create visualization overlays of the designs in situ. The overlay generated an AR environment at a certain level of immersion where the user was able to experience and engage with visualizations of the designs (i.e., the technology design had an active, immersive user experience, and they used motion tracking and immersive experience data within the construction walkthroughs. The platform included IMU sensors and a camera system for depth sensing. The AR application was developed according to the i-Tracker system architecture and used Tangoтм hardware (see Section 3.1).

Approach. The user interacted at a basic level by moving through the physical space (in situ) to perceive and engage in an emotional reaction to the physical location. The user movements were conceptualized as their walkthroughs in the physical space. While the user moved along the job site, motion-tracking data, to determine 
the user's position while moving freely within the limits of the physical area, was captured and mapped. The researchers arrived at random locations in the job site to ensure reliability of the data. The randomized selection captured available features on the job site for functional performance, including the way in which the features are computed and cached (e.g., the most salient features that could be identified for feature extraction-from objects like windows and doors - could be used for virtual visualization alignment).

\subsection{Evaluation}

An engaging user visualization interaction that enables natural responses to actions when performing the walkthroughs requires a certain level of component performance from the AR technology. The following are three different foci of AR technology performance that were chosen for evaluation - they are selected as a result of them being enabling technologies for satisfactory immersive experience: (1) motion-tracking accuracy; (2) motiontracking accuracy with area learning correction; and (3) adequate luminance levels.

Motion-tracking accuracy. Focused on measures of IMU sensor outputs without the use of visual sensing corrective methods, motion-tracking accuracy measures relate to the alignment of virtual content from both indoor and outdoor environments and determine how precisely $i$-Tracker hardware tracks the motion of a user. Tradeoffs of motion-tracking accuracy directly impact reliability.

Two tests, in which the computing device was moved back and forth between two known physical points, were initially performed. The first test (indoor test) had a variable distance ranging from $100 \mathrm{~cm}$ to $200 \mathrm{~cm}$ (see Figure 7). With 1 millimeter of precision, the mobile device was displaced over a sheet of graph paper 100 times to build a comparative baseline and add rigor and accuracy. This distance was a Euclidean distance and was used as a comparative baseline for the second test, which consisted of moving the mobile device between two known positions in the physical world. The baseline informed the level of accuracy through the difference (i.e., deviation from the baseline) between the tracked distance and the distance provided by the mobile platform. The second test included motions and activities of the typical walkthrough. Each test was executed 50 times to collect variances in the results and reduce the impact of intrinsic errors in data collection.

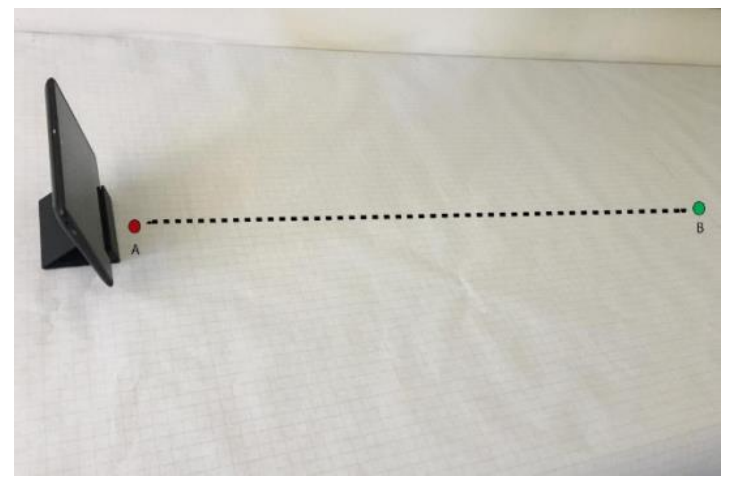

a) Experimental setup for testing the precision of $i$-Tracker's motion tracking.

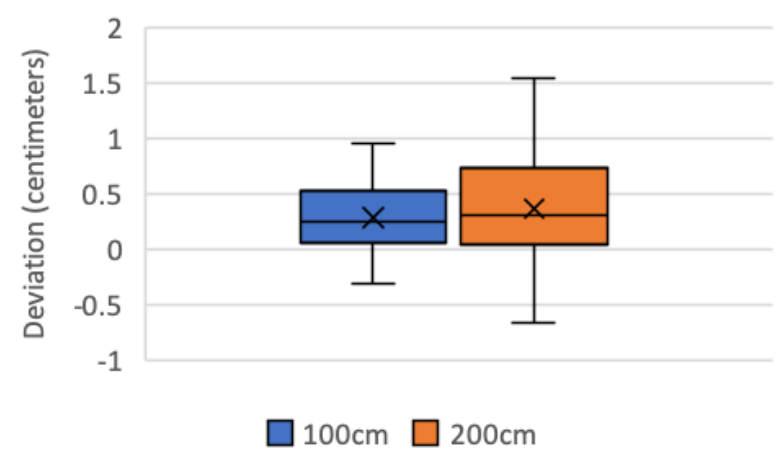

b) Motion-tracking error dispersion for precision analysis.

FIG. 7: Motion tracking experimental setup and results.

Figure 7 shows the experimental setup of the indoor experiment to test precision of the motion-tracking feature of the Tangoтм hardware on a small scale. The dotted line in the left figure shows the travel path of the $i$-Tracker unit (Tangотм hardware) to test the precision of the motion tracking. The right figure shows the mean and deviation of errors found during the experiment. The results show the error that the system accumulates during user motion between a starting point and endpoint. It was observed that the baseline displacements (output from the first test) have lower accumulation than the outdoor ones. Longer distances tend to attract more drift errors in the motiontracking estimation. Figure 8 shows examples of indoor and outdoor walkthrough settings, which emulates typical walkthroughs for construction sites. The different physical conditions of each setting have an impact on the functionality of technology devices (e.g., responses to sensing lighting conditions). 

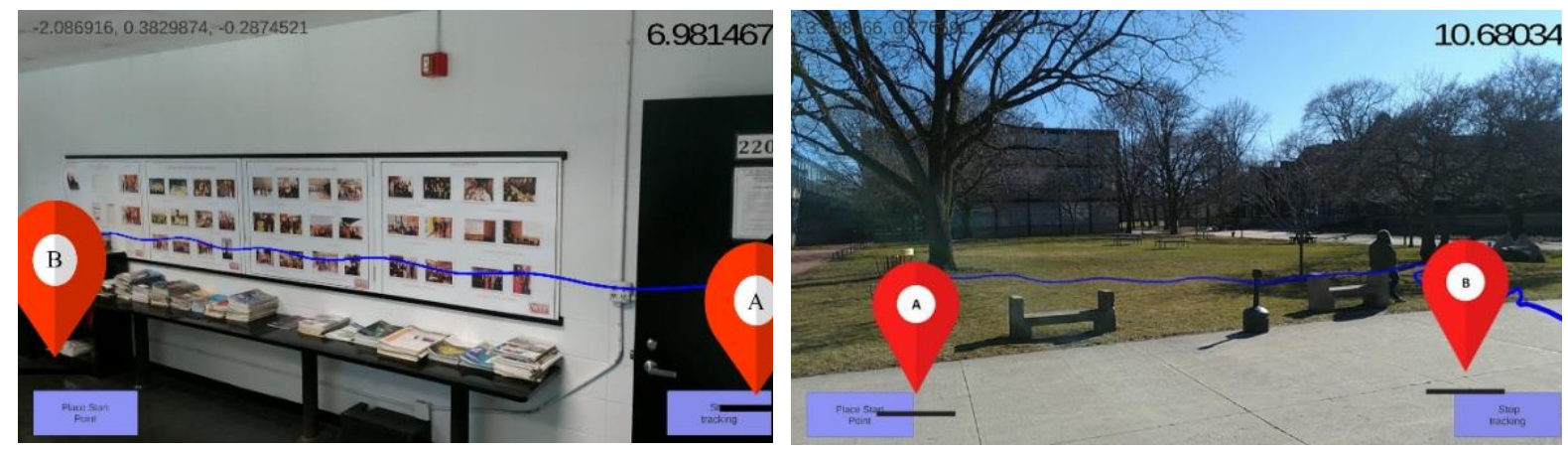

FIG. 8: Experimental walkthroughs of indoor (left) and outdoor (right) testing.

Motion-tracking accuracy with area learning correction. Experiments were performed, both indoor and outdoor, with an additional parameter for the implementation of an ADF. Taking advantage of area learning for the features of the mobile platform, a survey around the area was first executed, and the extracted features were stored in the corresponding ADF file. The ADF file was then loaded while performing the motion-tracking experiment. The ADF implementation showed significant improvements in accuracy compared to performance of the motion-tracking feature using IMU sensors. Figures 9 and 10 show the minimum, maximum, and mean errors generated by the system as well as an example of the standard deviation data for the baseline.

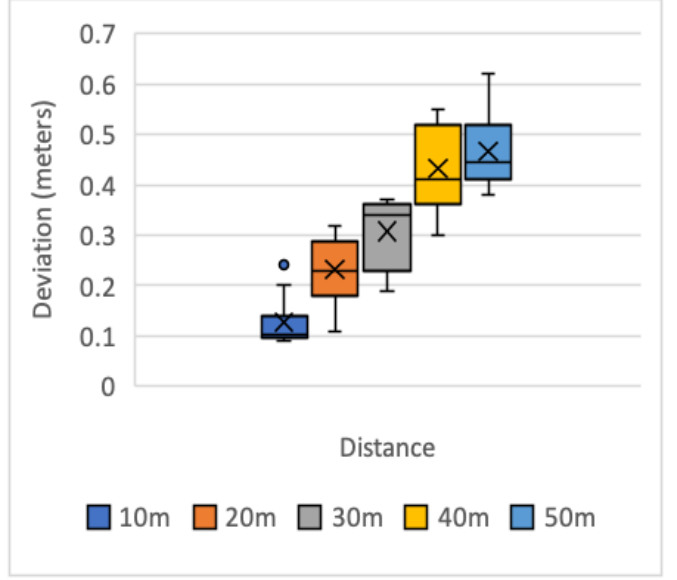

a) Motion-tracking error for the indoor environment.

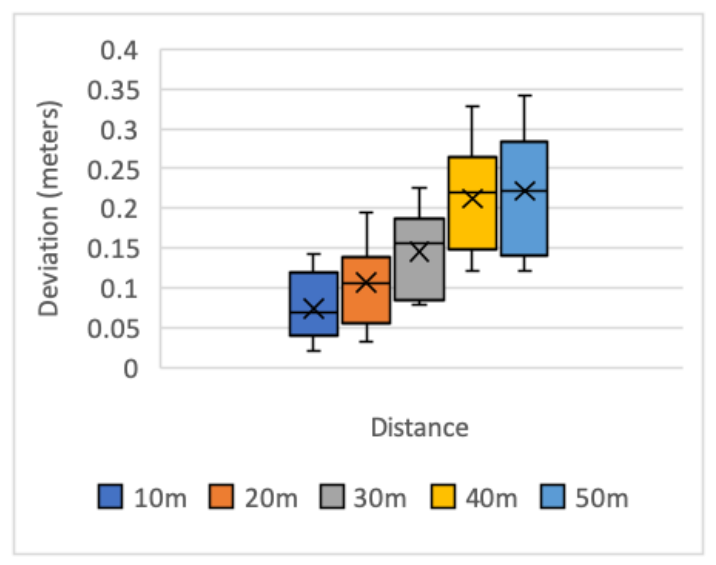

b) Error dispersion of motion tracking + ADF implementation for an indoor environment.

FIG. 9: Indoor motion-tracking accuracy deviations (errors) using IMU sensors and area learning correction.

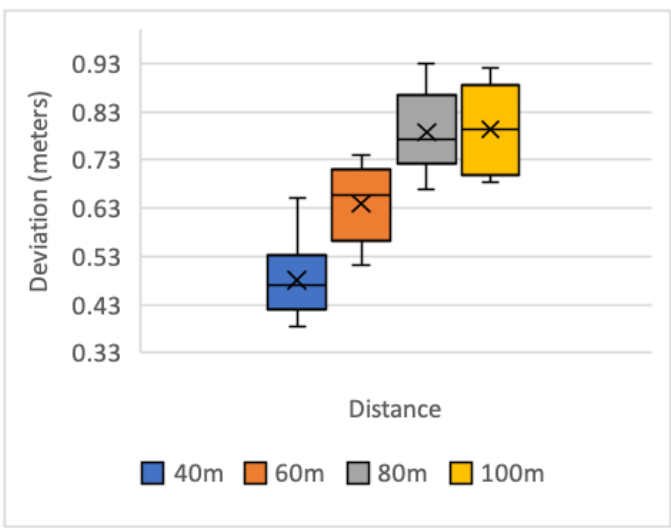

a) Motion-tracking error for the outdoor environment.

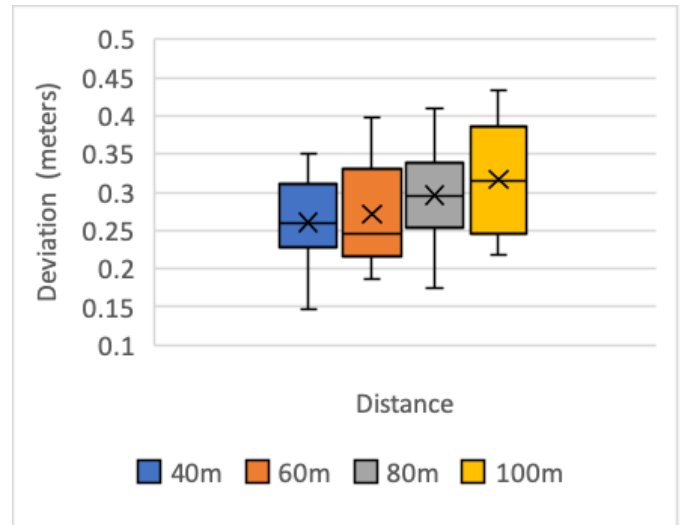

b) Error dispersion of motion tracking + ADF implementation for an outdoor environment.

FIG. 10: Outdoor motion-tracking accuracy (errors) with area learning correction feature. 
Table 1: Analysis of experimental findings of indoor and outdoor motion-tracking experiments.

\begin{tabular}{|l|l|l|l|l|l|}
\hline \multicolumn{2}{|c|}{} & $\begin{array}{l}\text { Standard } \\
\text { deviation }\end{array}$ & $\begin{array}{l}\text { Difference in } \\
\text { standard deviation }\end{array}$ & Mean error & $\begin{array}{l}\text { Decrease in } \\
\text { error }\end{array}$ \\
\hline \multirow{2}{*}{$\begin{array}{l}\text { Indoor } \\
\text { workspace }\end{array}$} & Motion tracking & 0.070 & 0.013 & 0.3130 & $51.40 \%$ \\
\cline { 2 - 3 } & Motion tracking + ADF & 0.057 & & 0.1521 & \\
\hline $\begin{array}{l}\text { Outdoor } \\
\text { workspace }\end{array}$ & Motion tracking & 0.083 & 0.014 & 0.6759 & $57.55 \%$ \\
\cline { 2 - 3 } & Motion tracking + ADF & 0.069 & & 0.2869 & \\
\hline
\end{tabular}

Luminance variation. Impacting performance of the AR technology and the realism of the scene as experienced by the user during walkthroughs, luminance variation was tested in the area learning feature by collecting data across various levels of natural light, ranging from a well-lit environment to a dark environment, as part of an outdoor, motion-tracking experiment. The experiment evaluated the dependency of the motion-tracking capability of the AR mobile computer platform on good lighting conditions or luminance levels. An ADF file was created for bright sunlight conditions in an outdoor environment. The "Sper Scientific toolkit" (Meters, 2019) was used to measure the luminance levels (see Figure 11). The data collected on accuracy measurements showed an inverse relationship with the measured luminance level. The findings are plotted in Figure 11.
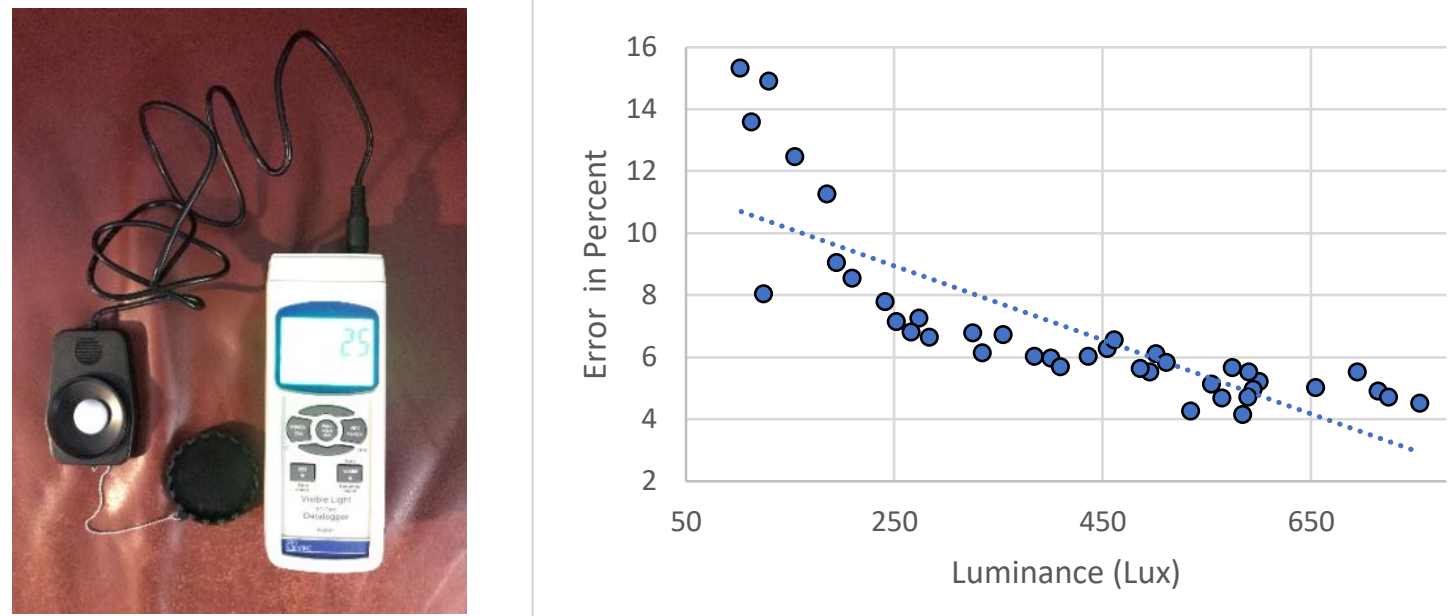

FIG. 11: Left, the luxmeter. Right, the inverse relation between drifting error and falling luminance levels.

\section{TRADE-OFF STRATEGIES FOR I-TRACKER TECHNOLOGY}

Pre-processing of the rendering of light and shadows and fragmentation of the BIM model are two trade-off strategies that are discussed in relation to limitations of the technology for an improved immersive experience.

Pre-processing of the rendering of light consists of estimating the effects of light on the game objects to add realism to the scene during the walkthrough by including lighting conditions. This involves reducing the effect of variable ambient lighting conditions by adjusting a lighting effect on the overlaid objects in real-time. The purpose was to constrain the system to run under ambient conditions to better accommodate reflectance from surfaces in the scene (e.g., incorporating the effect of reflections in the augmentations by a specific material and surface). The estimation of light data was stored in the texture files, which were later overlaid on top of the augmented objects to produce the visual effect. Unity3Dтм software was used to process the estimation and to add extra effects. Inconsistencies in lightning conditions were found to be reduced, thereby enhancing reliability and improving the user's immersive experience. This action enhanced the reliability of an augmented object by adding comfort and a more realistic view to the user.

Fragmentation of the BIM model consists of managing large-scale BIM models to build an augmented scene with minimum latency to meet limitations of the mobile device's processing capacity. Since the processing capacity of the mobile device limits the design and function of interactivity applications, this is considered a trade-off of 
performance with the interactivity of the AR application. A strategy was implemented based on the activation of the objects for the view on site. An activation system was built that changed the number of visible augmented objects based on the user location. In this way, it was possible to restrict data processing to only a few objects per scene. Lower latencies were achieved with approximately 100 objects for augmentation. Observed latency with a few seconds of delay appeared when there were more than 100 objects stored and activated in the mobile device. The effect was, while the users were moving along a walkthrough, a delay in alignment with respect to the physical world.

The use-case example presented tests of the i-Tracker technology applications for construction management activities in walkthroughs. Underpinned trade-off factors to the tests were aimed at responding to a more immersive experience by optimizing or balancing remote and local computation (performance), accuracy of the AR overlay (reliability), and latency of the AR and sensing features (functionality) (see Table 2). The focus was on achieving higher levels of immersion by matching visual experience with feedback from the user's bodysegment position and movement and by spatially orienting and accommodating visual and motor stimuli in the physical space. Evaluation of the technology features for the immersive experience was executed on the computing device in which the $i$-Tracker approach was deployed (Tangoтм).

Table 2. Actions and Trade-offs for quality of visualization and interaction in walkthroughs

\begin{tabular}{|c|c|c|c|}
\hline \multirow{2}{*}{$\begin{array}{l}\text { Performed Balancing } \\
\text { Actions in } \text { i-Tracker }\end{array}$} & \multicolumn{3}{|c|}{ Effects on Trade-off Factors } \\
\hline & Performance & Functionality & Reliability \\
\hline $\begin{array}{l}\text { Lower the number of BIM } \\
\text { objects to respond to user } \\
\text { demand }\end{array}$ & $\begin{array}{c}\text { Higher } \\
\text { (efficiency in } \\
\text { computational time) }\end{array}$ & $\begin{array}{l}\text { Lower } \\
\text { (latencies of the AR } \\
\text { visualization alignment } \\
\text { with respect with the } \\
\text { physical world) }\end{array}$ & $\begin{array}{c}\text { Lower } \\
\text { (availability of } \\
\text { information for an } \\
\text { immersive experience) }\end{array}$ \\
\hline Increase luminance & $\begin{array}{l}\text { Higher } \\
\text { (motion tracking- } \\
\text { capability) }\end{array}$ & $\begin{array}{l}\text { Lower } \\
\text { (latency in localization } \\
\text { and device pose in the } \\
\text { physical environment) }\end{array}$ & $\begin{array}{c}\text { Higher } \\
\text { (accuracy due reduction } \\
\text { of deviation errors) }\end{array}$ \\
\hline $\begin{array}{l}\text { Increase distance from the } \\
\text { origin with area learning } \\
\text { correction }\end{array}$ & $\begin{array}{c}\text { Lower } \\
\text { (responses of sensing } \\
\text { features) }\end{array}$ & $\begin{array}{l}\text { Higher } \\
\text { (responsiveness to the } \\
\text { user's demands for } \\
\text { walkthrough activities) }\end{array}$ & $\begin{array}{l}\text { Lower } \\
\text { (accuracy with the } \\
\text { alignments as drifting } \\
\text { error increases) }\end{array}$ \\
\hline
\end{tabular}

\section{DISCUSSION AND CONCLUSION}

Successful integration of AR technologies in walkthroughs to enable more seamless immersive experiences in construction management tasks poses significant challenges. An approach framed within trade-off factors (performance, functionality, and reliability) examined technology limitations and enabling features that respond to basic levels of immersion based on AR visual quality and technology-interaction for walkthroughs. A robust AR environment named "i-Tracker" was designed and developed as a technology platform for this examination in walkthroughs. The $i$-Tracker technology approach used area learning techniques to capture and process data for situational awareness by employing overlays of 3D engineering designs as an output. $i$-Tracker captured 3D features from the outdoor physical environment, registered the features within the AR system, and overlaid visualizations of design and data, thereby providing immersive and interactive features to the end user. The $i$ Tracker AR client was conceptualized to provide the users with increased awareness through immersive experiences, good quality of visualization, and interaction with the system.

The use-case example examined the performance, functionality, and reliability of $i$-Tracker technologies features to make awareness and immersion of the AR visualizations possible. Using a systematic approach, the use-case example demonstrated how the trade-off factors are balanced by imposing stringent constraints in some factors to achieve high demonstration in others. The performed actions on trade-off aimed to provide a satisfactory 
immersive AR experience in walkthroughs. It did so by achieving a continuous match of the AR overlay with feedback in an AR computer device monitoring the user's body-segment pose and movement in the physical space.

It was found that, as they produce ambiguity in visualization overlays for design interpretation, factors such as luminance and accuracy within the set of 3D features affected the AR immersive experience to a certain degree. Accuracy is critical for construction management tasks in walkthroughs. Walkthroughs encompass the stakeholders' (project engineers, architects, construction managers) multiple visits to an area of interest in the job site. The activity implies the user's change of location with considerable distances and different physical conditions from the origin. The user's change of location and variations of luminance directly impact accuracy. Superimposing visualizations of designs at a distance from the origin and from different physical conditions reduced the AR visualization quality, the state of awareness for design interpretation, and, ultimately, the outcomes of construction management tasks. The implication is a reduced ability to visualize designs using AR in activities that require high accuracy of location of the augmentations for their interpretation, such as in the case of project control tasks, where the expedient identification of deviations between the actual progress of construction and design is critical.

The AR location-tracking features of $i$-Tracker performed well in short distances of location change from the origin, but drifting errors accumulated as the changing of location distance from the origin increased. These errors reduce accuracy and thereby harmed the visualization and interaction quality in walkthroughs for an effective immersive experience. Drifting errors impeded matching AR overlays with feedback in the AR computer device registering the user's body-segment pose (arms and head) during the user's movement in the physical space. These errors reduced the ability to accommodate (synchronize) visual and motor modalities in the physical space. Luminance also impacted the performance of i-Tracker technology. Higher drifting errors (deviations) were experienced in indoor environments with lower luminance than in outdoor environments with higher luminance.

To empower project stakeholders and all job-site personnel to incorporate project management activities in a more compelling immersive experience, it is critical to address location-tracking limitations of technology. Experiments focused on motion-tracking feature evaluation, which employs mobile computer devices for AR applications (Tangотм), would enable readers to understand the effect of the trade-off factors when similar use cases are deployed with AR technology. Future research should focus on the effects of actions on the trade-off factors with new improvements in sensing and computing capabilities technology.

For future developments, the $i$-Tracker approach will focus on reducing the impact of offloading to achieve higher augmentation accuracy and reduced latency of visualizations of designs. For example, working with large BIM models involves a considerable number of 3D objects, which would increase the overall size of the offloaded application and the associated processing time. Efficient trade-offs could be made with a cloud service to store considerable resources and back-end processing to reduce latency in the mobile device, in lieu of fragmenting the BIM for extensive walkthroughs. New technologies will significantly impact the dynamics of these trade-offs. 5G networks, for example, will provide new bandwidth capabilities to redesign approaches for a resource-intensive computational task and efficient computing (offloading versus mobile computing) for minimum latency. New ARCore and ARkit technologies would provide better augmentation accuracy by incorporating depth-sensing cameras and IMU sensors. The next iteration of $i$-Tracker will capitalize on these advancements to fine-tune the trade-off factors for a more efficient immersive experience to fully realize the potential AR technology features in Architecture, Engineering, and, Construction (AEC) practices.

In summary, the authors envision that AR technology research will be a focus on trade-off factors to benefit immersive experiences in walkthroughs. Many trade-offs for performance, functionality, and reliability need to be investigated, ranging from optimization of remote versus local computation of designs to computing latency, communication delays, and limitations on the robustness of computing and network systems. Outcomes of the evaluation of i-Tracker will give insights - in terms of motion tracking capabilities, methods of improving accuracy due to drifting errors and heightening responsiveness to user's demands - on how to improve successes of AR technology integration into immersive in situ experiences to, therefore, positively and significantly impact AEC practices. 


\section{REFERENCES}

Aggarwal, J. K., \& Cai, Q. (1999). Human motion analysis: A review. Computer vision and image understanding, 73(3), 428-440.

Alsafouri, S., \& Ayer Steven, K. (2019). Mobile Augmented Reality to Influence Design and Constructability Review Sessions. Journal of Architectural Engineering, 25(3), 04019016. doi:10.1061/(ASCE)AE.1943-5568.0000362

Anandan, P. (1989). A computational framework and an algorithm for the measurement of visual motion. International Journal of Computer Vision, 2(3), 283-310.

Antonenko, P., \& Mutis, I. (2017). Using Unmanned Aerial Systems to Bring STEM Field Experiences to the Classroom. Paper presented at the National Association for Research in Science Teaching (NARST), San Antonio, TX. http://www.narst.org/annualconference/2017conference.cfm

Azuma, R., Baillot, Y., Behringer, R., Feiner, S., Julier, S., \& MacIntyre, B. (2001). Recent Advances in Augmented Reality. IEEE Comput. Graph. Appl., 21(6), 34-47. doi:10.1109/38.963459

Azuma, R. T. (1997). A survey of augmented reality. Presence: Teleoperators \& Virtual Environments, 6(4), 355-385.

Barfield, W., \& Caudell, T. (Eds.). (2001). Fundamentals of Wearable Computers and Augumented Reality. CRC Press.

Behzadan, A. H., \& Kamat, V. R. (2006, June, 14-16, 2006). Animation of Construction Activities in Outdoor Augmented Reality. Paper presented at the Joint International Conference on Computing and Decision Making in Civil and Building Engineering, Montreal, Canada.

Davison, A. J., Cid, Y. G., \& Kita, N. (2004). Real-time 3D SLAM with wide-angle vision. IFAC Proceedings Volumes, 37(8), 868-873.

Elbamby, M. S., Perfecto, C., Bennis, M., \& Doppler, K. (2018). Toward low-latency and ultra-reliable virtual reality. IEEE Network, 32(2), 78-84.

Google Project Tango. (2015). Project Tango Technology -. Retrieved from https://www.google.com/atap/project-tango/

Green, K. C., \& Gilbert, S. W. (1995). Great expectations: Content, communications, productivity, and the role of information technology in higher education. Change: The magazine of higher learning, 27(2), 8-18.

Hou, L., Wang, X., \& Truijens, M. (2013). Using Augmented Reality to Facilitate Piping Assembly: An ExperimentBased Evaluation. Journal of Computing In Civil Engineering. doi:10.1061/(asce)cp.1943-5487.0000344

Jianbo, S., \& Tomasi, C. (2000). Good features to track. Paper presented at the IEEE Computer Society Conference on Computer Vision and Pattern Recognition, Hilton Head Island, SC.

Jonassen, D. H. (2011). Learning to solve problems : a handbook for designing problem-solving learning environments. New York: Routledge.

Klatzky, R. L., Wu, B., \& Stetten, G. (2008). Spatial Representations From Perception and Cognitive Mediation: The Case of Ultrasound. Current Directions in Psychological Science, 17(6), 359-364. doi:10.1111/j.14678721.2008.00606.x

Koch, C., Neges, M., König, M., \& Abramovici, M. (2014). Natural markers for augmented reality-based indoor navigation and facility maintenance. Automation in Construction, 48, 18-30. doi:10.1016/j.autcon.2014.08.009

Kovachev, D., Yu, T., \& Klamma, R. (2012). Adaptive computation offloading from mobile devices into the cloud. Paper presented at the IEEE 10th International Symposium on Parallel and Distributed Processing with Applications, Shanghai, China.

Li, W., Nee, A., \& Ong, S. (2017). A State-of-the-Art Review of Augmented Reality in Engineering Analysis and Simulation. Multimodal Technologies and Interaction, 1(3). doi:10.3390/mti1030017

Li, X., Yi, W., Chi, H.-L., Wang, X., \& Chan, A. P. C. (2018). A critical review of virtual and augmented reality (VR/AR) applications in construction safety. Automation in Construction, 86, 150-162. doi:10.1016/j.autcon.2017.11.003

Marr, D., Poggio, T., \& Ullman, S. (1979). Bandpass channels, zero-crossings, and early visual information processing. 69(6), 914-916. doi:10.1364/JOSA.69.000914

Meters, S. S. (2019). PH, Conductivity, TDS, ORP, Temperature, Humidity. Retrieved from https://www.sperdirect.com/

Milgram, P., \& Kishino, F. (1994). A taxonomy of mixed reality visual displays. IEICE Transactions on Information and Systems, 77(12), 1321-1329.

Mutis, I. (2018). Spatial-Temporal Cognitive Ability: Coupling Representations to Situations and Contexts for Coordinating Activities. In I. Mutis, R. Fruchter, \& C. C. Menassa (Eds.), Transforming engineering education. Innovative Computer-Mediated Learning Technologies (pp. 12): ASCE. 
Mutis, I., Fruchter, R., \& Menassa, C. C. (2018). Transforming Engineering Education: Innovative Computer-Mediated Learning Technologies. Reston, Virginia: American Society of Civil Engineers- ASCE.

Mutis, I., \& Issa, R. R. A. (2014). Enhancing spatial and temporal cognitive ability in construction education through augmented reality and artificial visualizations. Paper presented at the Computing in Civil and Building Engineering Workshop, Orlando, Fl.

Papagiannakis, G., Singh, G., \& Magnenat-Thalmann, N. (2008). A survey of mobile and wireless technologies for augmented reality systems. Computer Animation and Virtual Worlds, 19(1), 3-22.

Park, C.-S., \& Kim, H.-J. (2013). A framework for construction safety management and visualization system. Automation in Construction, 33, 95-103. doi:10.1016/j.autcon.2012.09.012

Park, C.-S., Lee, D.-Y., Kwon, O.-S., \& Wang, X. (2013). A framework for proactive construction defect management using BIM, augmented reality and ontology-based data collection template. Automation in Construction, 33, 6171.

Rankohi, S., \& Waugh, L. (2013). Review and analysis of augmented reality literature for construction industry. Visualization in Engineering, 1(1), 9. doi:10.1186/2213-7459-1-9

Shelton, B. E. (2003). How Augmented Reality Helps Students Learn Dynamic Spatial Relationships. (Doctor of Philosophy Dissertation). College of Education, Retrieved from http://itls.usu.edu/ bshelton/resources/dissertation_bes_final.pdf

Shi, J., \& Tomasi, C. (2000, June). Good Features to Track. Paper presented at the IEEE Computer Society Conference on Computer Vision and Pattern Recognition, Hilton Head Island, SC.

Shirazi, A., \& Behzadan Amir, H. (2015). Design and Assessment of a Mobile Augmented Reality-Based Information Delivery Tool for Construction and Civil Engineering Curriculum. Journal of Professional Issues in Engineering Education and Practice, 141(3), 04014012. doi:10.1061/(ASCE)EI.1943-5541.0000229

Tang, A., Owen, C., Biocca, F., \& Mou, W. (2003). Comparative effectiveness of augmented reality in object assembly. Paper presented at the Proceedings of the SIGCHI Conference on Human Factors in Computing Systems, Ft. Lauderdale, Florida, USA.

Unity Technologies. (2018). A cross-platform game engine. Retrieved from https://unity3d.com/

van Krevelen, D. W. F., \& Poelman, R. (2010). A Survey of Augmented Reality Technologies, Applications and Limitations. The International Journal of Virtual Reality, 9(2), 1-20. doi:citeulike-article-id:8213624

Wang, P., Wu, P., Wang, J., Chi, H. L., \& Wang, X. (2018). A Critical Review of the Use of Virtual Reality in Construction Engineering Education and Training. Int $J$ Environ Res Public Health, 15(6). doi:10.3390/ijerph15061204

Wang, X., \& Dunston, P. S. (2006). Compatibility issues in Augmented Reality systems for AEC: An experimental prototype study. Automation in Construction, 15(3), 314-326. doi:10.1016/j.autcon.2005.06.002

Wang, X., Kim, M. J., Love, P. E. D., \& Kang, S.-C. (2013). Augmented Reality in built environment: Classification and implications for future research. Automation in Construction, 32, 1-13. doi:https://doi.org/10.1016/j.autcon.2012.11.021

Woo, J. H. (2007). BIM (building information modeling) and pedagogical challenges. Paper presented at the Proceedings of the 43rd ASC National Annual Conference, Northern Arizona University.

Zaher, M., Greenwood, D., \& Marzouk, M. (2018). Mobile augmented reality applications for construction projects. Construction Innovation. doi:10.1108/ci-02-2017-0013

Zhou, Y., Luo, H., \& Yang, Y. (2017). Implementation of augmented reality for segment displacement inspection during tunneling construction. Automation in Construction, 82, 112-121. doi:10.1016/j.autcon.2017.02.007

Zollmann, S., Hoppe, C., Kluckner, S., Poglitsch, C., Bischof, H., \& Reitmayr, G. (2014). Augmented Reality for Construction Site Monitoring and Documentation. Proceedings of the IEEE, 102(2), 137-154. doi:10.1109/JPROC.2013.2294314 